\title{
What's new for the clinician? Summaries of and excerpts from recently published papers
}

SADJ October 2017, Vol 72 no 9 p433 - p436

Compiled and edited by $\mathbf{V}$ Yengopal

\section{Capping carious exposures in adults: a randomized controlled trial investigating mineral trioxide aggregate versus calcium hydroxide}

\begin{abstract}
Kundzina R, Stangvaltaite L, Eriksen HM, Kerosuo E. International Endodontic Journal 2017; 50: 924-32.
\end{abstract}

Direct pulp capping is a method designed to preserve pulp vitality through hard tissue repair of the open exposure, and studies have shown it to be superior to root canal treatment in the long run, provided the patient is a young adult and there is no proximal exposure. ${ }^{1}$ For many decades, calcium hydroxide has been the standard material for maintaining pulp vitality. Both clinically and histologically it has been found to produce satisfactory results in indirect and direct pulp capping, because it is capable of stimulating the formation of tertiary dentin by the pulp. In contact with vital pulp tissue it contributes to the formation of reparative dentin, a special variant of tertiary dentin, which seals exposures by newly formed hard tissue. Nevertheless, calcium hydroxide has some drawbacks. Poor bonding to dentin, material resorption and mechanical instability are among them. As a result, calcium hydroxide does not prevent microleakage in the long run. The porosities ('tunnel defects') of the newly formed hard tissue may act as a portal of entry for microorganisms. These may cause secondary inflammation of the pulp tissue and are thought to be responsible for failed maintenance of tooth vitality. In addition, the high $\mathrm{pH}$ (12.5) of calcium hydroxide suspensions causes liquefaction necrosis at the surface of the pulp tissue.

MTA is an endodontic cement that is extremely biocompatible, capable of stimulating healing and osteogenesis, and is hydrophilic. MTA is a powder that consists of fine trioxides (tricalcium oxide, silicate oxide, bismute oxide) and other hydrophilic particles (tricalcium silicate and tricalcium aluminate, responsible for the chemical and physical properties of this aggregate), which set in the presence of moisture. Hydration of the powder results in formation of a colloidal gel with a $\mathrm{pH}$ of 12.5. The gel solidifies to a hard solid in approximately 3 to 4 hours. This cement is different from other materials currently in use because of its biocompatibility, antibacterial properties,

Jeff Yengopal: BChD, MChD (Community Dentistry) Stellenbosch Department of Community Dentistry, School of Oral Health Sciences,

University of the Witwatersrand. E-mail: Veerasamy.Yengopal@wits.ac.za.

\author{
ACRONYMS \\ $\mathrm{CH}$ : Calcium hydroxide group \\ MTA: Mineral trioxide aggregate
}

marginal adaptation and sealing properties, and its hydrophilic nature. ${ }^{1}$

Kundzina and colleagues from Norway (2017) 1 reported on a multicentre, parallel-group RCT that sought to compare the effectiveness of MTA and calcium hydroxide as a direct pulp capping (DPC) material in adult molars with carious pulpal exposure.

\section{MATERIALS AND METHODS}

This study was a randomized, controlled, parallel, patientblinded, two-arm superiority trial with a 1:1 allocation ratio. The inclusion criteria were applied as follows: age 18-55 years; first or second permanent molar with a proximal carious lesion (primary or secondary caries); no history of pain or the presence of pain indicating, at most, reversible pulpitis; positive response to a cold test or to electric pulp testing according to the routine choice of each clinic, bitewing radiograph showing a carious lesion in at least the inner $1 / 3$ of the dentine; periapical radiograph showing closed apex and normal periapex (with no radiolucency or widening of the periodontal ligament space); attachment loss not exceeding $4 \mathrm{~mm}$; noncontributory medical history (including pregnancy); and no use of medication (no antibiotics during the previous month). Only one pulp cap was to be included per subject. In cases where a subject had several eligible molars, the one with the deepest lesion was chosen. Exclusion criteria included a lack of pulpal exposure after complete removal of the caries and failure to control the bleeding in exposed pulp within $10 \mathrm{~min}$.

The clinical procedures were standardized. Briefly, the tooth was anaesthetized and isolated with a rubber dam, and the caries was removed using a complete excavation strategy. The cavity outline (Class II) was cut down to sound enamel using a high-speed bur and water cooling. With a round bur at low speed, the carious dentine was then completely removed from the non-pulpal walls of the 
cavity until the dentine was found to be hard when checked with a sharp probe and all cavity margins were inside the sound tooth structure. To ensure good prerequisites for the sealability of the margins of the cavity, a caries detector dye was applied. On the pulpal wall(s), only a hand excavator was used to remove the caries until no or little dye staining was present. In cases of pulpal exposure, the bleeding was controlled within 10 min using cotton pellets soaked in buffered $0.5 \% \mathrm{NaOCl}$

Patients were randomized using the envelope method, with block sizes 4-6-4-6, with each study clinic acting as a separate allocation unit. Envelopes were sent to the study clinics in batches of 20 . The envelope revealed the treatment group to which the subject was allocated and was opened only after bleeding of the pulp was successfully controlled. Patients were not informed of their allocation group.

In the Calcium hydroxide $(\mathrm{CH})$ group, a thin layer of a commercial liner (Dycal ${ }^{\circledR}$ ) was applied to the pulpal exposure and left to set. In the MTA group, white ProRoot ${ }^{\circledR}$ powder was mixed according to the manufacturer's instructions and a 2-mm-thick layer was placed directly over the pulpal exposure and the surrounding dentine, leaving at least 2 $\mathrm{mm}$ of dentine and enamel available circumferentially for the bonded composite restoration. After placement of the MTA, a flat, water-moistened cotton pellet was placed directly over the material. As the setting of the MTA needed to be checked after 1 week, the cavities in both groups then received a temporary filling (Fuji IX glass-ionomer cement). After 1 week, the entire temporary filling was removed for patients in the MTA group, and the hardness of the MTA was verified with a dental probe. In the $\mathrm{CH}$ group, part of the temporary filling was left under the permanent filling. Any postoperative pain was recorded, pulpal status was checked applying a cold or electric pulp testing, and if there were no symptoms, the cavity was permanently restored with a composite resin material used at the study clinic.

The primary outcome was the survival of capped pulps. Survival was defined as a non-symptomatic tooth that responded to sensibility testing and did not exhibit any periapical changes radiographically. Follow-up included pulpal testing and periapical radiograph at six, 12, 24 and 36 months. The secondary outcome measure was postoperative pain one week after treatment. The patients were asked whether they had any pain or had experienced it during the first three days.

\section{RESULTS}

Eighty patients were recruited into this trial. Ten subjects were excluded due to a lack of pulpal exposure after the complete removal of caries. Consequently, 70 subjects were randomly allocated to either the $\mathrm{CH}$ group $(n=37$; mean age 30.9 years SD: 9.9) or the MTA group ( $n=33$; mean age 30.2 SD 9.7). The study was closed when all patients had received at least 36 months of follow-up, had the capped pulp fail or had been lost to follow-up.

The Kaplan-Meier survival analysis showed an 85\% cumulative survival rate of pulps in the MTA group and $52 \%$ in the $\mathrm{CH}$ group. This difference was significant $(P=0.006)$ according to the log-rank test. The effect size was 0.25 , and the number needed to treat was three $(N N T=3)$.
The subanalyses showed no significant difference (log-rank chi-square $=0.323 ; P=0.570$ ) in the cumulative survival rates between into-the-pulp and not-into-the pulp groups, 0.58 and 0.71 , respectively.

Almost half of the subjects (34/70) had experienced preoperative pain at baseline that was not more severe than reversible pulpitis; these subjects were equally distributed between the two material groups. Only 18 of the 70 subjects reported postoperative pain at 1 week. Regardless of the presence of preoperative pain, there were no significant associations between the material and postoperative pain, although postoperative pain was observed slightly more often (10/33) in the MTA group than in the $\mathrm{CH}$ group (8/37). No adverse effects were reported in either group.

\section{CONCLUSION}

The authors concluded that Mineral trioxide aggregate (MTA) was more effective than conventional $\mathrm{CH}$ dressing as a direct pulp capping material in molars with carious pulpal exposure in adult patients.

\section{IMPLICATIONS FOR PRACTICE}

The superior material properties and clinical performance of MTA makes it an ideal choice for use in endodontics.

\section{Reference}

1. Kundzina R, Stangvaltaite L, Eriksen HM, Kerosuo E. Capping carious exposures in adults: a randomized controlled trial investigating mineral trioxide aggregate versus calcium hydroxide. International Endodontic Journal 2017; 50: 924-932. 


\section{Evaluation of pit-and-fissure sealants placed with four different bonding protocols: a randomized clinical trial}

\begin{abstract}
Khare M, Shrikrishna SB, Ramya S, Rao A. International Journal of Paediatric Dentistry 2017; 27: 444-53.
\end{abstract}

The use of pit and fissure sealants has become accepted as an effective intervention for the prevention of occlusal caries in the molar teeth of young children. The evidence for the clinical efficacy and cost-effectiveness of sealants in reducing occlusal caries in molars has been highlighted in recent papers.1 Resin-based sealant materials are most commonly used and are regarded as the 'gold standard' for sealing pits and fissures. Their cariespreventive effect relies on the sealing of pits and fissures through micro-retention, created through tags after acid etching of enamel. However, these are easily destroyed by saliva contamination, reducing micro-retention and consequently, the caries-preventive effect. 1 Moreover, the preventive benefits and resin-based sealant retention are gained and maintained only as long as the sealants remain completely intact and bonded in place

The application of adhesive during sealant placement which allows optimal infiltration and encapsulation of inter- and intra-prismatic boundaries of etched enamel and the formation of long adhesive tags has been studied. The dehydrating activity of solvents in the adhesive such as acetone or ethanol displaces water from the deep fissure walls and allows deeper penetration of adhesive and sealant resin. This has been shown to increase bond strength, reduce micro-leakage, and improve shortterm clinical success. Both etch-and-rinse (total etch) type and self-etch type of bonding agents have been studied. Multimode one-bottle universal adhesives have been developed recently to make the clinical procedure more user-friendly. These new adhesives can be used as self-etch or as etch-and-rinse adhesives. This enables the clinician to selectively acid etch enamel and bond to enamel in etch-and-rinse mode and dentin in selfetch mode with the additional advantage of chemical bonding to enamel and dentin. Khare and colleagues from India reported on a RCT that sought to evaluate the effectiveness of pit-and-fissure sealants applied to teeth using etch-and-rinse, self-etch, and multimode adhesives during bonding of pit-and-fissure sealants.

The null hypothesis stated was that there is no difference in sealant retention, fissure caries incidence, and marginal discoloration after use of self-etch or etch-and-rinse or multimode adhesive in the bonding protocol compared with conventional bonding protocol of pit-and-fissure sealant.

\section{MATERIALS AND METHODS}

This was a split mouth, randomized, double-blinded clinical trial. Children aged 6-10 years with all four erupted, non-carious first permanent molars which were completely erupted through the gingiva, with deep pits and fissures and DMFT/deft 1 or more with satisfactory cooperative behaviour (Frankl score 3 or 4 ) and oral hygiene (OHI-S $\leq 3)$ were selected. Patients with history of any medical disease or long-term medication that might interfere with the study were excluded. Patients with history of abnormal parafunctional activity, under fluoride application regimen, restorations on first permanent molars, and history of allergies to resins were also excluded from the study.

Finally, 52 patients with a total of 208 first permanent molars, four in each patient, were included in the study. They were randomly assigned into four groups using block allocation (block size 4) by an operator not involved in other aspects of the study. Randomization was performed using a random table of numbers. Details of the allocated group were recorded on cards contained in sequentially numbered, opaque, sealed envelopes.

Sealants were placed according to one of the following bonding protocols:

- Group 1: Conventional acid etching without adhesive (control group)

- Group 2: Etch-and-rinse adhesive (Adper Single Bond 2)

- Group 3: Multimode universal adhesive (Scotch-bond Universal Adhesive) used in etch-and-rinse mode

- Group 4: Self-etch adhesive (G-BOND) used after acid etching

Clinical sealing protocol

For the sealant placement, all procedures were standardized. Moisture control was maintained by cotton rolls and saliva ejector. All applications were carried out by a single operator. The sealants were placed in the grooves of occlusal surfaces of permanent first molars (including the buccal groove of lower molars and palatal groove of upper molars). Sealants were applied on the permanent molars with one of the bonding protocols:

- Conventional acid etching without adhesive. Etchant was applied using a micro-applicator brush, left for 15 seconds, and then washed with water spray for 10 seconds. The tooth surface was dried by cotton blotting.

- Etch-and-rinse adhesive (Adper Single Bond2). Etchant was applied using a micro-applicator brush, left for 15 seconds, and was thereafter washed with water spray for $10 \mathrm{~s}$. The tooth surface was dried by cotton blotting. The bonding agent was applied using the micro-applicator for 15 seconds and spread evenly using air spray for 5 seconds. The bonding agent was then light cured for 10 seconds.

- Multimode universal adhesive used in etch-and-rinse mode (Scotch-bond Universal Adhesive). Etchant was applied using a micro-applicator and left for 15 seconds and was thereafter washed with water spray for 10 seconds. The tooth surface was dried by cotton blotting. The bonding agent was applied using the micro-applicator and rubbed for 20 seconds and then air-dried for 5 seconds. The bonding agent was then light cured for 10 seconds.

- Self-etch adhesive used after acid etching (G-BOND). Etchant was applied using a micro-applicator and left for 15 seconds and was thereafter washed with water 
spray for 10 seconds. The tooth surface was dried by cotton blotting. The bonding agent was applied to the etched fissures and left undisturbed for 10 seconds and then gently air-dried for 5 seconds. The bonding agent was then light cured for 10 seconds.

After the respective adhesive application, the resin sealant (Clinpro ${ }^{\mathrm{TM}}$ Sealant) was applied to the pits and fissures and gently teased through the fissure with the tip of a periodontal probe to prevent voids and air entrapment. Then, the applied fissure sealants were polymerized using a LED curing light for 20 seconds. The cured sealant was checked for voids using an explorer, which were refilled. All the sealant applications were cross-checked using mouth mirror and explorer by an experienced examiner. The occlusion was checked with articulation paper. Finishing and polishing was performed using one-step composite polishers (Shofu) at low speed. The participants were unaware of the type of bonding protocol selected for each tooth.

Children were scheduled for evaluation visits at 3, 6, and 12 months from the date of treatment. Sealant retention was recorded according as: A) complete retention, B) partial loss (B1, without caries; B2, with caries), C) complete loss (C1, without caries; C2, with caries). Loss of enamel translucency along the margins, softness at the base of exposed fissures, and defects along the margins with discoloration were denoted as dental caries. Marginal discoloration to any degree was recorded as present/absent.

Examination for all teeth was carried out by a single calibrated examiner who was unaware of group allocation. Teeth with partial/complete loss of sealants were repaired by sealant application, and caries was treated with appropriate restorations. Such teeth were subsequently dropped from the study.

\section{RESULTS}

The study sample consisted of 32 males and 20 females. The mean age of the participants was $8.69 \pm 0.98$ years. The age distribution of the sample was as follows: 6 years old (3.8\%), 7 years old (3.8\%), 8 years old (32.7\%), 9 years old (38.5\%), and 10 years old (21.2\%). Of 52 participants, three participants did not report for follow-up due to illness at 6-month follow-up and one participant did not report at 12-month follow-up due to migration to another city. This amounted to a drop-out rate of $7.69 \%$. For the final analysis of the data, the dropouts and teeth with partial/ complete loss of sealant were eliminated and the rest of the data was considered.

At the end of three months, the number of teeth with completely retained sealants was highest in Group 2 followed by Group 3, Group 4, and Group 1. After one year, complete retention was highest in Group 2 and 3 followed by Group 4 and Group 1. Most of the sealant loss was of partial type with only five teeth showing complete loss. Etch-and-rinse group showed the least partial loss followed by universal and self-etch groups. Caries was observed only in one tooth which belonged to Group 3 at three months.

A nonparametric Friedman analysis was carried out for intragroup (within group) comparison of sealant retention rate at three, six, and 12 months, which revealed statistical significant difference over time in etch-and-rinse group $(P=0.018)$. There was no statistical difference in sealant retention rate among other groups with time $(P=0.368$ for Group 1; P $=0.135$ for both Group 3 and Group 4). Group 2 had the highest complete sealant retention at three months $(92.3 \%)$ which decreased to $91.8 \%$ at six months. But by 12 months, only $77.1 \%$ of sealants had been retained with greatest loss occurring in the six to 12-month period. Kruskal-Wallis test was used for intergroup (between group) comparison of sealant retention at three, six and 12 months. At the three-month follow-up, the difference between the groups was found to be statistically significant $(P=0.002)$ but was not significant at both six-month and 12-month follow-up ( $\mathrm{P}=0.119$ and 0.952, respectively). Mann-Whitney U-test after Bonferroni correction revealed that there was a statistically significant difference between Group 1 (conventional acid etch) and Group 2 (etch and rinse) at three-month follow-up.

The pattern of marginal discoloration, an early indicator of loss of marginal integrity, showed similar trends as sealant retention. Intergroup comparison of marginal discoloration using chi-square test revealed statistically significant difference at three months but no significant difference at six months and 12 months. Intragroup comparisons over time at three, six and 12-month follow-up using chi-square test revealed significant difference in the etch-and-rinse group $(P=0.02)$ but not in other groups $(P>0.05)$. Pearson's correlation revealed that there was no statistically significant relation between OHIS as well as DMFT/deft scores with sealant retention rate thus confirming that the level of oral hygiene and dental caries of the participants did not affect the sealant retention rate. Sealant retention was higher in maxillary molars as compared with mandibular molars at all follow-up visits, but the difference was not statistically significant $(P=0.31)$.

\section{CONCLUSIONS}

Under the conditions of this study, the authors found that the use of self-etch or etch-and-rinse or multimode adhesive during pit-and-fissure sealant application does not significantly enhance sealant retention nor decrease marginal discoloration at 12-month follow-up. Etch-andrinse adhesive is advantageous only at short-term threemonth follow-up.

\section{IMPLICATIONS FOR PRACTICE}

This trial found that the retention of sealants did not significantly increase with use of adhesives. Hence, it may not be necessary to routinely use dental adhesives for bonding pit-and-fissure sealants, taking into consideration the increase in complexity and cost of the procedure.

\section{Reference}

1. Khare M, Shrikrishna SB, Ramya S, Rao A. Evaluation of pit-and-fissure sealants placed with four different bonding protocols: a randomized clinical trial. International Journal of Paediatric Dentistry 2017; 27: 444-453. 